\title{
Remedial robot-assisted laparoscopic surgery in the treatment of neuropathic inguinodynia and repair of inguinal hernia
}

\author{
Mohamed Mohamed*, Mohamed Elsouri, Adel Elmoghrabi, Daniel Coffey, Michael McCann \\ Hurley Medical Center, Trauma Services Department, Michigan State University, United States
}

Received: August 28, 2016

Accepted: September 23, $2016 \quad$ Online Published: October 14, 2016

DOI: $10.5430 /$ css.v2n4p54

URL: http://dx.doi.org/10.5430/css.v2n4p54

\begin{abstract}
Introduction: Inguinodynia or persistent groin pain, is a common postoperative complication of inguinal hernia repair that may interfere with patients' daily activities. Remedial surgery is often required for management. Based on our review of literature, the use of robotically assisted laparoscopy as an option for remedial surgery appears nonexistent.

Case presentation: A 54-year-old male patient with a past history of inguinal hernia repair four years ago presented with severe, burning right inguinal pain that peaked 6 months prior to presentation. History was positive for failed multiple analgesic treatment options. Examination revealed tenderness over the right external ring and a prior right inguinal surgical incision with paresthesia along the distribution of the right ilioinguinal nerve. Based on the history and CT findings, the patient was diagnosed with meshoma and ilioinguinal nerve entrapment. Remedial posterior robotic laparoscopic surgery combined with ilioinguinal neurectomy was performed, resulting in complete resolution of pain post-operatively.

Conclusions: In our case of post-inguinal hernia repair of neuropathic inguinodynia, laparoscopic robot-assisted surgery combined with ilioinguinal neurectomy led to excellent results.
\end{abstract}

Key Words: Inguinodynia, Inguinal hernia repair, Robotic, Neurectomy, Ilioinguinal, Entrapment

\section{INTRODUCTION}

Inguinodynia, or persistent groin pain lasting at least three months after surgery, has surpassed recurrence as the most common postoperative complication of inguinal hernia repair. ${ }^{[1]}$ Prevalence rates for inguinodynia after an open or laparoscopic hernia repair are highly variable and range from $1 \%-63 \%,{ }^{[1]}$ with an estimated $12 \%-25 \%$ of these patients experiencing pain that interferes with their daily activities. ${ }^{[2,3]}$ Remedial surgery is often required for management. We present a combined robotic and open approach for the management of inguinodynia in a 54-year-old male suffering from persistent groin pain four years after an open inguinal hernia repair. To the best of our knowledge, this represents the first reported use of robotic surgery in the course of treating inguinodynia.

\section{Case presentation}

A 54-year-old male patient presented to the outpatient clinic with severe, burning right inguinal pain rated at $10 / 10$ on a numeric pain scale. The pain increased with physical exertion and interfered with his ability to perform house chores such as mowing the lawn and shoveling snow. It initially started

\footnotetext{
*Correspondence: Mohamed Mohamed; Email: mmohame4@hurleymc.com; Address: Departamento de Computacao, Centro Federal de Educação Tecnológica de Minas Gerais, Av. Amazonas, 7675, Zip Code: 30.510-000, Belo Horizonte, Brazil.
} 
four years ago after a right-sided plug and patch inguinal hernia repair and continued with an intermittent course, peaking six months prior to the current presentation. History was positive for seeking multiple analgesic treatments, including local nerve blocks, and was unclear for a prior surgical procedure involving the ilioinguinal nerve performed for pain relief (questionable neurectomy) 8 months ago. Nonetheless, there was failure of complete pain resolution, which led the patient to seek our medical attention. Other previous surgeries included hiatal and umbilical hernia repair. Past medical \& family histories were unremarkable.

On examination, vital signs were normal. Abdominal examination revealed severe tenderness over the right external ring and mild tenderness over the prior right inguinal surgical incision. Neurological examination revealed paresthesia along the distribution of the right ilioinguinal nerve. Results of a CT of the abdomen and pelvis without contrast performed at an outside facility were reviewed (the details of which were not available at time of writing this report), and in combination with the history, the patient was provisionally diagnosed with a meshoma and likely entrapment of the ilioinguinal nerve. Due to the prolonged history of pain and prior surgeries, the patient was planned for meshoma excision and re-implantation of a new mesh via posterior robot-assisted laparoscopic surgery (Transabdominal Preperitoneal Repair TAPP) for higher operative precision in order to minimize tissue dissection, and achieve a quicker recovery. Additionally, concomitant prophylactic revision of the prior questionable ilioinguinal neurectomy via an anterior approach was considered to ensure complete pain relief. However, the decision was deferred until findings of the posterior approach were evaluated intraoperatively.

Via a supraumbilical incision, the posterior laparoscopic robotic approach was performed with dissection of the posterior aspect of the right groin. The mesh plug was identified and found to be irregular (measuring $3 \mathrm{~cm} \times 2 \mathrm{~cm} \times 1.2 \mathrm{~cm}$ ) and secured with permanent Prolene sutures that entrapped the ilioinguinal nerve. The sutures and mesh plug were precisely removed, and replaced by replaced by a 3 inch $\times 5$ inch Bard ${ }^{\circledR}$ mesh and secured via absorbable Vicryl and Monocryl sutures. Due to the entrapping mesh and sutures, anterior visualization of the ilioinguinal nerve via an anterior approach was decided upon intraoperatively in order to assess the status quo of the nerve, especially in the face of questionable history of ilioinguinal neurectomy. Anterior exposure revealed that the nerve was still intact and an ilioinguinal neurectomy was completed.

Post-operative histopathological examination of the excised nerve and meshoma revealed nerve fibrosis, confirming the

Published by Sciedu Press diagnosis of nerve entrapment, and was unremarkable for the meshoma. The postoperative course was uneventful and the patient was discharged on postoperative day 1. After a one-year follow up, the patient is free of pain, living his normal life-style and able to resume his prior activities.

\section{Discussion}

Based on our review of literature, the use of robot-assisted laparoscopy as an option for remedial surgery appears nonexistent. However as described in our case report, this option can lead to effective treatment and complete resolution of neuropathic inguinodynia.

Robotic laparoscopic surgery offers the surgeon increased precision and accuracy for easier dissection. The robotic platform used for our case was the Da Vinci machine. As opposed to the traditional laparoscopic approach offering 2-dimentional (2D) visualization, the Da Vinci machine offers 3D visualization. ${ }^{[4]}$ The latter is achieved via two $5 \mathrm{~mm}$ scopes within a $12 \mathrm{~mm}$ telescope projecting onto two screens that synchronize into a single, binocular view for the surgeon. ${ }^{[5]}$ This creates a true 3D experience for the surgeon because the right and left images are kept separate resulting in each eye having its own visual field. Unlike the traditional laparoscopic approach, the arms of the robot have seven degrees of freedom and four degrees of axial rotation. ${ }^{[5]}$ This type of dexterity closely mimics the human hand and allows for "wrist movements". These characteristics made this an excellent approach for meshoma excision as precision is necessary for the small and sensitive post-hernia repair inguinal region. Based on the origin of pain, inguinodynia can be classified into: non-neuropathic and neuropathic. Pain in non-neuropathic inguinodynia results from excessive scar formation from a reaction with a prosthetic mesh or other foreign body, while in neuropathic inguinodynia, it is from direct damage to the inguinal nerve. ${ }^{[3]}$ Nerve entrapment is usually the underlying cause of damage in neuropathic inguinodynia occurring during inguinal hernia repair. In our case, the patient's meshoma and Prolene sutures entrapped the ilioinguinal nerve causing neuropathy. Other susceptible inguinal nerves include the iliohypogastric and genitofemoral nerves. ${ }^{[6]}$ The resulting neuropathic pain usually develops in the sensory distribution of the damaged inguinal nerve(s) and can lead to crippling pain. ${ }^{[3]}$ In a retrospective review of 225 patients who underwent surgery for both types of inguinodynia, 72 of the 100 patients diagnosed with neuropathic inguinodynia experienced nerve entrapment by suture, staple, or mesh. ${ }^{[2,3]}$ Thus, prevention of nerve entrapment during the initial inguinal hernia repair is crucial and should be considered as the first line of defense against neuropathic inguinodynia. There are two surgical approaches for hernia 
repair: open and laparoscopic. There is no consensus on the specific approach type that leads to a lower incidence of nerve entrapment. This is displayed in a meta-analysis of 17 randomized studies that compared patients undergoing inguinal hernia repair using either of the two approaches. ${ }^{[7]}$ In patients who underwent repair using the open versus laparoscopic approach, the incidence of inguinodynia was higher in eight and lower in four of the studies. In the remaining five studies, no differences were appreciated with either of the approach types. A laparoscopic posterior approach is often utilized when a patient has a history of open anterior hernia repair. ${ }^{[8]}$ This approach enables the avoidance of fibrotic scar tissue from the previous operation- which is more difficult to operate through - while allowing access to deeply placed meshes. Further, repeated surgery in the same location is associated with increased hospital length of stay, pain, and recurrence of hernia. ${ }^{[8]}$ The disadvantage of such an approach is the need for specialized surgeon training and the increased operation costs. In our case, due to the prior history of hernia repair and the reduced risks, the posterior approach was selected as the method of repair over an anterior approach.

Treatment for inguinodynia caused by nerve entrapment during hernia repair may be categorized into: preventative and remedial, with the latter being sub-divided into surgical and non-surgical. Preventative measures are intended to avoid future complications for the patient. Meanwhile, remedial measures rectify problems that either occur inevitably, or as a complication of prior hernia repair.

Preventative measures begin with adequate identification of all three inguinal nerves (ilioinguinal, iliohypogastric, and genitofemoral) during surgery, and the use of appropriate mesh and sutures types during repair. Knowledge of the inguinal neuroanatomy and meticulous preservation of the three inguinal nerves is believed to reduce the risk of nerve damage. ${ }^{[1,6,9]}$ Additionally, using the appropriate mesh type and fixation materials during the initial hernia repair have also been shown to decrease the chance of foreign body reaction, nerve entrapment, and chronic pain. ${ }^{[9]}$ Using absorbable sutures, as undertaken in our case, and reducing the number of tacks or sutures used to fixate a mesh have been shown to reduce the incidence of inguinodynia by preventing nerve entrapment. ${ }^{[7,10]}$ Another preventative measure for inguinodynia due to nerve entrapment is prophylactic neurectomy of the inguinal nerves susceptible to injury during the initial hernia repair. ${ }^{[11]}$ In a randomized prospective study, patients that underwent open inguinal hernia repair followed by an ilioinguinal nerve neurectomy reported significantly less pain after follow-up for 180 days compared to patients who did not receive the neurectomy. ${ }^{[9]}$ This study, along with many others, demonstrates that prophylactic excision of inguinal nerves after an open repair decreases the incidence of neuropathic inguinodynia. ${ }^{[2,9,11]}$ The main challenge in performing a neurectomy is deciding on the specific nerve for excision. A triple neurectomy of the ilioinguinal, iliohypogastric and genitofemoral nerves is accepted as the most effective treatment option for neuropathic inguinodynia. ${ }^{[6]}$ In a retrospective study of 225 patients with inguinodynia, a triple neurectomy led to complete pain relief in $80 \%$ of patients one month after surgery. In our patient, pain was localized to the sensory distribution of the ilioinguinal nerve, hence our decision to perform an ilioinguinal neurectomy only. For a neurectomy to be effective, the nerve must be resected as proximally and distally as possible. ${ }^{[12]}$ In our case, the additional anterior approach was necessary to fully visualize the ilioinguinal nerve and ultimately determine if the nerve was truly excised during the patient's prior hernia repair. Ultimately, the nerve appeared intact and was resected appropriately. Despite the compelling findings in studies demonstrating successful outcomes with prophylactic neurectomy following open hernia repair, it still remains a decision primarily based on both surgeon and patient consensus. ${ }^{[13]}$ It is important to note that in our case, having the concomitant posterior intraabdominal approach performed may have exposed the patient to the potential risks of robot-assisted laparoscopic surgery during hernia repair. Recurrent posterior hernia repair risks damage to testicular blood vessels, nerves, and lymphatics with the potential of local hematomas; however, these risks are similar to an anterior approach, but with reduced incidence. ${ }^{[8]}$ Other potential risks may include port site infection, inadvertent bowel injury, injury to femoral/iliac vessels, etc. None of these risks however, were encountered in our case.

When preventative measures are deemed unsuccessful, remedial non-surgical and surgical treatments for neuropathic inguinodynia become necessary. The most common nonsurgical management of neuropathic inguinodynia is the use of analgesics, particularly, local inguinal nerve blocks. Nerve blocks interfere with neural transmission and can temporarily reduce neuralgia, serving therapeutic and diagnostic capabilities. ${ }^{[1]}$ Nonetheless, there is paucity in the literature on success rates of nerve blocks as a treatment option for neuropathic inguinodynia. ${ }^{[3,10]}$ Further, their temporary effect and requirement for multiple administrations represents a drawback of their use as a therapeutic modality. ${ }^{[10]}$ Despite being an attractive diagnostic tool in deciding which inguinal nerve to excise in a surgical neurectomy, their diagnostic capacity is limited. Heise and Starling demonstrated no correlation between a positive response to a nerve block and the effectiveness of a following neurectomy. ${ }^{[14]}$ Similarly was the case with our patient. Persistent post-neurectomy pain can result from a variety of surgical complications, including 
excision of the incorrect nerve, suture entrapment, partial division, crushing, or diathermy of the other non-excised nerves. ${ }^{[15]}$ For example, nerve blocks administered to wrong or unintended nerves could result in a misleading diagnosis (if the pain dissipates) leading to the wrong nerve or branch being excised with resultant persistence of pain postoperatively. ${ }^{[6]}$ This may have been the case in our patient.

Remedial surgery represents the last line of defense against neuropathic inguinodynia. Surgical intervention usually involves groin exploration, foreign body removal, and neurectomy. In a cases like ours, where the mesh and materials used for its affixation entrap an inguinal nerve, removal of the mesh and/or the affixation material can lead to pain relief. Sampath et al. demonstrated complete pain relief in two patients with neuropathic pain after removing a single staple in one, and part of a mesh in the other. ${ }^{[16]}$ However, foreign body removal alone does not always lead to favorable

\section{REFERENCES}

[1] Lange JF, Kaufmann R, Wijsmuller AR, et al. An international consensus algorithm for management of chronic postoperative inguinal pain. Hernia. 2015; 19(1): 33-43. PMid: 25138620. http: //dx.doi.org/10.1007/s10029-014-1292-y

[2] Aasvang E, Kehlet H. Chronic postoperative pain: the case of inguinal herniorrhaphy. Br J Anaesth. 2005; 95(1): 69-76. PMid: 15531621. http://dx.doi.org/10.1093/bja/aei019

[3] Hakeem A, Shanmugam V. Inguinodynia following Lichtenstein tension-free hernia repair: a review. World J Gastroenterol. 2011; 17(14): 1791-6. PMid: 21528050. http://dx.doi.org/10.3748 /wjg.v17.i14.1791

[4] Escobar PF, Haber GP, Kaouk J, et al. Single-port surgery: laboratory experience with the daVinci single-site platform. JSLS. 2011; 15(2): 136-41. PMid: 21902962. http://dx.doi .org/10.4293/10868 $0811 \mathrm{X} 13022985132128$

[5] Kalan S, Chauhan S, Coelho RF, et al. History of robotic surgery. Journal of Robotic Surgery. 2010; 4(3): 141-7. PMid: 27638753. http://dx.doi.org/10.1007/s11701-010-0202-2

[6] Bjurstrom MF, Nicol AL, Amid PK, et al. Pain control following inguinal herniorrhaphy: current perspectives. J Pain Res. 2014; 7: 277-90. PMid: 24920934.

[7] Poobalan AS, Bruce J, Smith WC, et al. A review of chronic pain after inguinal herniorrhaphy. Clin J Pain. 2003; 19(1): 48-54. PMid: 12514456. http://dx.doi.org/10.1097/00002508-2003010 00-00006

[8] Karatepe O, Acet E, Altiok M, et al. Preperitoneal repair (open posterior approach) for recurrent inguinal hernias previously treated with Lichtenstein tension-free hernioplasty. Hippokratia. 2010; 14(2): 119-21. PMid: 20596268. results as it might fail to address irreversible nerve damage that may have been established, which can continue to be a source of neuropathic pain. ${ }^{[1]}$ Heise et al. demonstrated that mesh removal plus neurectomy led to pain relief in $62 \%$ of chronic inguinodynia patients. ${ }^{[14]}$ Ultimately, the results in our patient after combined mesh replacement and ilioingual neurectomy, were excellent.

\section{Conclusions}

This case illustrates potential in laparoscopic robot-assisted surgery as a remedial treatment option for the neuropathic inguinodynia in patients with prior inguinal hernia repair, complicated by meshoma and ilioinguinal nerve entrapment. Concomitant ilioinguinal neurectomy in our case led to complete resolution of chronic inguinodynia.

\section{CONFLICTS OF INTEREST Disclosure}

The authors declare they have no conflicts of interest.
[9] Shamita C, Rohit K. A comparative study of inguinodynia following lichtenstein hernioplasty with or without elective neurectomy of ilioinguinal nerve. Hellenic Journal of Surgery. 2014; 86(3): 137-41. http://dx.doi.org/10.1007/s13126-014-0115-z

[10] Aroori S, Spence RA. Chronic pain after hernia surgery-an informed consent issue. Ulster Med J. 2007; 76(3): 136-40. PMid: 17853638.

[11] Mui WL, Ng CS, Fung TM, et al. Prophylactic ilioinguinal neurectomy in open inguinal hernia repair: a double-blind randomized controlled trial. Annals of surgery. 2006; 244(1): 27-33. PMid: 16794386. http://dx.doi.org/10.1097/01.sla.0000 $217691.81562 .7 \mathrm{e}$

[12] Campanelli G, Bertocchi V, Cavalli M, et al. Surgical treatment of chronic pain after inguinal hernia repair. Hernia. 2013; 17(3): $347-$ 53. PMid: 23519769. http://dx.doi.org/10.1007/s10029-0 13-1059-x

[13] Murovic JA, Kim DH, Tiel RL, et al. Surgical management of 10 genitofemoral neuralgias at the Louisiana State University Health Sciences Center. Neurosurgery. 2005; 56(2): 298-303. PMid: 15670378. http://dx.doi.org/10.1227/01.NEU.0000 148000.04592.E1

[14] Heise CP, Starling JR. Mesh inguinodynia: a new clinical syndrome after inguinal herniorrhaphy? J Am Coll Surg. 1998; 187(5): 514-8. http://dx.doi.org/10.1016/S1072-7515(98)00215-4

[15] Khoshmohabat H, Panahi F, Alvandi AA, et al. Effect of Ilioinguinal Neurectomy on Chronic Pain following Herniorrhaphy. Trauma Mon. 2012; 17(3): 323-8. PMid: 24350117. http://dx.doi.org/10. 5812/traumamon. 6581

[16] Sampath P, Yeo CJ, Campbell JN. Nerve injury associated with laparoscopic inguinal herniorrhaphy. Surgery. 1995; 118(5): 829-33. http://dx.doi.org/10.1016/S0039-6060(05) 80272-7 